\title{
Qualität und Qualitätsmessung in der Langzeitpflege aus Sicht der Nutzerinnen und Nutzer
}

\author{
Pia-Theresa Sonntag, Nadja-Raphaela Baer, Adelheid Kuhlmey, Ralf Suhr \\ und Liane Schenk \\ (c) Der/die Autor(en) 2018 \\ K. Jacobs et al. (Hrsg.), Pflege-Report 2018 \\ https://doi.org/10.1007/978-3-662-56822-4_9
}

\section{Zusammenfassung}

Eine transparente Qualitätsbeurteilung und -berichterstattung in der stationären Langzeitpflege setzt voraus, die Perspektiven aller relevanten Akteure zu berücksichtigen. Neben der Sicht der Experten ist dabei auch die der Nutzerinnen und Nutzer maßgeblich. Dieser Beitrag geht der Frage nach, was Pflegequalität aus Sicht von Pflegebedürftigen und ihren Angehörigen bedeutet. Auf Grundlage der aktuellen Forschungslage wird zunächst herausgearbeitet, inwiefern die Nutzerperspektive bei der Pflegequalitätsmessung bislang berücksichtigt wird. Im Mittelpunkt stehen ausgewählte Ergebnisse der vom Institut für Medizinische Soziologie und Rehabilitationswissenschaft der Charité durchgeführten und vom AOK-Bundesverband und dem Zentrum für Qualität in der Pflege (ZQP) geförderten Studie zur »Weiterentwicklung der Qualitätsberichterstattung in der Langzeitpflege«. Dabei werden zum einen die Qualitätsbeurteilungen von Pflegebedürftigen und deren Angehörigen und zum anderen die von ambulant und stationär versorgten Pflegebedürftigen identifiziert und vergleichend analysiert. Der Fokus liegt hierbei auf den Präferenzen in Bezug auf Qualitätsbereiche und -kriterien, die aus Sicht der verschiedenen Nutzergruppen entscheidend für die Auswahl einer adäquaten Pflegeeinrichtung sind. Während sich beim Subgruppenvergleich zwischen Angehörigen und Pflegebedürftigen nur punktuelle Differenzen herausstellen, differieren die Präferenzen der ambulant und stationär Versorgten deutlich.

In order to assure a transparent quality assessment and reporting in long-term care, the consideration of multiple perspectives is required. More specifically, not only the experts' perspectives, but also those of the users are relevant. This paper deals with the quality of long-term care from the users' points of view, i.e. from the perspectives of people in need of long-term care as well as the perspectives of their relatives. Encapsulating the latest research, this paper firstly addresses to what extent the users' perspective has been considered within the context of quality assessment and assurance in long-term care so far. The focus is on selected findings from the study "Enhancing Quality Reports in Long-Term Care" conducted by the Institut für Medizinische Soziologie und Rehabilitationswissenschaft of the Charité and funded by the AOK-Bundesverband and the Zentrum für Qualität in der Pflege $(Z Q P)$. Quality assessments of care-dependent people on the one hand and those of their relatives on the other hand have been identified and subjected to a comparative analysis. Moreover, the quality evaluations of the subgroups »outpatients « and »inpatients " were contrasted. Central to this investigation was the identification of user-specific preferences with regard to quality fields and quality criteria which play a decisive role in the choice of an adequate long-term care facility. While the subgroup analysis of people in need of long-term care and their relatives revealed only little differences, the preferences of inpatients and outpatients vary considerably. 


\subsection{Relevanz der Nutzerperspektive für die Pflegequalität}

\section{- Bedeutung eines nutzerorientierten}

\section{Qualitätsverständnisses}

Der Umzug in eine Pflegeinstitution wird von den Betroffenen als einschneidendes Ereignis erlebt, das u. a. mit einem Autonomieverlust verbunden ist. Die Verarbeitung dieses kritischen Lebensereignisses gelingt umso eher, wenn der Heimeintritt bewusst vorbereitet wird (vgl. Walter 1995; Klingenfeld 1999). Eine aktive Gestaltung des Heimeintritts wird aber nicht zuletzt durch den Mangel an einer bedarfsgerechten und nutzerfreundlichen Präsentation bestehender Pflegeangebote erschwert. Das schließt eine transparente Darstellung der Qualität der einzelnen Pflegeeinrichtungen ein. Eine solche Transparenz setzt voraus, dass neben der Expertensicht die Perspektive der Nutzerinnen und Nutzer, ihre Wünsche und Bedarfe einbezogen werden, damit sie eine informierte Auswahl treffen können. Bekannt ist, dass wissenschaftliche oder professionelle Definitionen und Bewertungen von Pflegequalität von jenen der Nutzerinnen und Nutzer abweichen können (vgl. Mittnacht 2010). In diesem Zusammenhang weist Bolz (2016) darauf hin, dass das Erfahrungswissen der Nutzerinnen und Nutzer von grundlegender Bedeutung für eine ganzheitliche Qualitätsbeurteilung sei. Die Dimension »Erfahrung « ist der Expertenperspektive nicht inhärent, prägt aber Erwartungen an und Bewertungen von Pflegequalität entscheidend mit (vgl. Bolz 2016; Duffy et al. 2001). Eine ganzheitliche Qualitätsbeurteilung setzt also die explizite und differenzierte Berücksichtigung beider Sichtweisen voraus (vgl. Duffy et al. 2001; Kämmer 2008; Roth 2002).

Moderne Konzepte zur Pflegequalität berücksichtigen diese Komplexität und Multidimensionalität (vgl. Hasseler 2014). So konstatiert Hasseler über die Anforderungen an eine systematische Qualitätsmessung und -berichterstattung in der Langzeitpflege, »dass für die Messung und Beurteilung von Qualität in der pflegerischen Versorgung ein dynamisches und systemisches Qualitätsverständnis erforderlich ist, das die soziale, räumliche, materielle, gesellschaftliche und institutionelle Umwelt einbezieht « (Hasseler 2014, S. 71). Wenn die pflegerische Versorgung als dynamischer Aushand- lungsprozess zwischen den verschiedenen Leistungserbringern sowie den Pflegebedürftigen und ihren Angehörigen (vgl. Donabedian 1966) konzeptualisiert wird, ist eine subjektorientierte Betrachtung von Pflegequalität möglich (vgl. Mittnacht 2010; Kämmer 2008, 2015). Kämmer (2015) spricht sich für ein solches Verständnis des Qualitätsbegriffs aus, das die »Lebenswelten« Pflegebedürftiger ins Blickfeld nimmt und dabei deren persönliche Erfahrungswerte, akute Bedürfnisse und Wünsche beachtet. Für das pflegerische Handeln bedeutet dies, »eine Umwelt für pflegebedürftige Menschen zu schaffen, in der die Gestaltung eines ganz normalen, an der eigenen, individuell passenden und gewohnten Lebensrealität orientierten Alltags gefördert wird « (Kämmer 2015, S. 40).

\section{- Nutzerorientierte Qualitätsberichterstattung und -darstellung}

Die bestehende Qualitätsberichterstattung in der Langzeitpflege gerät in die Kritik, weil sie die Nutzerperspektive nur unzureichend berücksichtigt (vgl. Geraedts et al. 2012; Josat et al. 2006; Bolz 2016; Stemmer und Arnold 2014). Mit Blick auf die von Wingenfeld et al. (2011) entwickelten IndikatorenSets zur Beurteilung der Ergebnisqualität in der stationären Altenhilfe kritisieren Stemmer und Arnold (2014) insbesondere die ergebnisfokussierte Qualitätsberichterstattung, die von dem tatsächlichen Informationsbedarf der Nutzerinnen und Nutzer sowie deren primärer Orientierung an strukturellen und prozessualen Qualitätskriterien abweicht. Die Autoren warnen vor der Vernachlässigung der Nutzerperspektive, da sich die einseitige Bezugnahme auf die Sicht des Fachpersonals in »defizitorientierten Indikatoren manifestieren " und somit die "genuine Zielgröße [des Pflegesystems], ... personenzentrierte Förderung bzw. Aufrechterhaltung von Lebensqualität « (Stemmer und Arnold 2014, S. 104) verfehlen könnte.

Die Bedeutung einer individuellen, bedürfnisorientierten Versorgung für die Pflegequalität wird in diversen (inter-)nationalen Arbeiten hervorgehoben (vgl. Geraedts et al. 2012; Bolz 2016; Schenk et al. 2013; Josat et al. 2006; Rantz et al. 1999). So bezeichnen Geraedts et al. (2012) die bis dato hintangestellte Perspektive der Nutzerinnen und Nutzer als "größtes Problem der Qualitätsbewertung" (Ge- 
raedts et al. 2012, S. 156). Josat et al. (2006) betonen die Relevanz der Nutzerperspektive aus ethischnormativer Sicht, der zufolge die Berücksichtigung der individuellen Bedürfnisse von Pflegebedürftigen essentiell für eine menschenwürdige pflegerische Versorgung sei. Hasseler (2014) beklagt den Mangel an systematischen Erkenntnissen bezüglich des nutzerspezifischen Informationsbedarfs über stationäre Pflegeangebote und verweist darauf, dass für eine fundierte Entscheidungsfindung eine nutzeradjustierten Informationsvermittlung notwendig sei. Die spezifischen Informationsbedarfe verschiedener Nutzergruppen (z. B. hochbetagte Pflegebedürftige, Nutzerinnen und Nutzer mit Migrationshintergrund) sowie unterschiedliche Verbraucherinteressen müssen bei der Präsentation der Angebote berücksichtigt werden (Hibbard und Peters 2003). In dem von der Bertelsmann Stiftung 2017 innerhalb des Projekts »Weiße Liste« herausgegebenen »Reformkonzepts zur verbraucherorientierten Qualitätsberichterstattung in der Pflege" wird das Ziel der Optimierung von »Public Reportings« im Sinne der Qualitätstransparenz fokussiert. Die Autoren Strotbek et al. (2017) plädieren für eine übersichtliche und nutzerorientierte Darstellung von Pflegequalität. Ein so ausgerichtetes Public Reporting soll der Definition von Pflegequalität aus Verbrauchersicht gerecht werden und vier Qualitätsebenen einbeziehen: (1) Gesundheitsbezogene Pflegequalität, (2) Betreuungs- und Aktivierungsqualität, (3) Nutzerbezogene Struktur- und Prozessqualität sowie (4) das Erfahrungswissen von Bewohnern, Angehörigen sowie des Personals.

\section{- Relevante Qualitätskriterien aus Sicht der Nutzerinnen und Nutzer}

Strotbek et al. (2017) befragten Nutzerinnen und Nutzer der "Weißen Liste« u. a. nach ihrer Zufriedenheit mit den Informationen zu Pflegeanbietern und ermittelten eine Inkongruenz zwischen dem aktuellen Informationsangebot und dem Informationsinteresse der Nutzerinnen und Nutzer. Diese sehen sich insbesondere bezüglich der von ihnen als besonders wichtig empfundenen Auswahlkriterien unzureichend informiert, wie etwa hinsichtlich personalbezogener Kriterien, zu welchen beispielweise »die Freundlichkeit des Personals" oder »ein würde- und respektvoller Umgang« zählen.
Geraedts et al. (2012) veröffentlichten im Gesundheitsmonitor 2011 eine Rangliste der zehn am häufigsten als "wichtig« beurteilten Aspekte bei der Wahl einer Pflegeeinrichtung. Dabei zeigte sich, dass aus Verbrauchersicht personalbezogene Kriterien, die sich insbesondere auf die persönliche Zuwendung beziehen (z. B. respektvoller Umgang mit den Pflegebedürftigen), und versorgungsbezogene Qualitätskriterien (z. B. ausreichend Zeit für die Pflege) von größerer Bedeutung für die Auswahl eines Pflegeheims sind. Weitere Untersuchungen zeigen, dass Pflegequalität für Betroffene vorwiegend subjektives Wohlbefinden der Pflegebedürftigen, intersubjektive Beziehungen sowie interpersonale und kommunikative Kompetenzen des Pflegepersonals bedeutet (vgl. IfD Allensbach 2009; Josat et al. 2006; Mittnacht 2010; Hasseler 2014). Lebensqualität ist ein weiteres bedeutendes Qualitätskriterium für Nutzerinnen und Nutzer (vgl. Stemmer und Arnold 2014; Strotbek et al. 2017). So konstatieren Strotbek et al. (2017), dass neben der gesundheitsbezogenen Pflegequalität die subjektiv empfundene Lebensqualität ein zentrales Motiv Pflegebedürftiger für die Auswahl einer Pflegeeinrichtung darstellt. Internationale Forschungsergebnisse bestärken die deutschen Studien. Beispielsweise eruieren Rantz et al. (1999) in ihrer US-amerikanischen qualitativ-explorativen Studie sechs Qualitätsdimensionen für die stationäre Altenpflege: Personal, pflegerische Versorgung, Familieneinbindung, Kommunikation, Umgebung und Einrichtung, heimische Atmosphäre und Kosten. Dabei sind die Dimensionen "pflegerische Versorgung « und »Personal « von besonderer Bedeutung für die Qualitätsbeurteilung der Pflege aus Nutzersicht (vgl. Rantz et al. 1999, vgl. Sonntag et al. 2017).

\section{- Differenzierte Qualitätskriterien von Pflegebedürftigen und Angehörigen}

Bei der Suche, Evaluation sowie Auswahl einer geeigneten Pflegeeinrichtung spielen Angehörige der Pflegebedürftigen eine zentrale Rolle. Sie haben häufig einen großen Einfluss auf den Entscheidungsprozess (vgl. Kelle et al. 2014; Nübling et al. 2004; Roth 2002) und bleiben »ein wichtiges Bindeglied zwischen der früheren Lebenswelt und dem neuen Lebensumfeld der Pflegebewohnerinnen und -bewohner. Dabei sind unterschiedliche Interessen, 
Selbstverständnisse und Gefühle der Bewohnerinnen und Bewohner und ihrer Angehörigen zu berücksichtigen [...]« (Engels und Pfeuffer 2007, S. 15). Zur Gewährleistung der Pflegequalität ist also neben der Anpassung der Versorgung an die pflegebedürftige Person selbst auch die Orientierung des Pflegeprozesses an den Wünschen, Bedürfnissen und der Expertise derer, die sie bei der Entscheidung unterstützen, von weitreichender Bedeutung. Bei der Qualitätsberichterstattung werden die Perspektiven unterschiedlicher Nutzergruppen nur wenig berücksichtigt. Einige Studien beleuchten die unterschiedlichen Bedarfe und Wünsche von pflegebedürftigen Personen und Angehörigen differenzierter (vgl. Josat 2005; Josat et al. 2006; Nübling et al. 2004; Roth 2002). Josat (2005) kommt u. a. zu dem Ergebnis, dass »Angehörige eine klare Trennung zwischen Qualitätskriterien, die für sie selbst wichtig sind, und solchen, die sie für die Bewohner als wichtig erachten [vornehmen] «(Josat 2005, S. 173). Beispielsweise stellt sich aus Sicht der befragten Angehörigen das Kriterium »Wohlfühlen des Bewohners/der Bewohnerin « als das Wichtigste heraus (Josat 2005). Darüber hinaus verdeutlicht eine Literaturanalyse von 13 internationalen Forschungsarbeiten die divergierende Präferenzsetzung verschiedener Qualitätskriterien von Pflegeheimbewohnerinnen und -bewohner sowie Angehörigen. Pflegebedürftige erachten zumeist jene Qualitätskriterien als besonders wichtig, die sich auf die autonome Lebensführung und selbstständige Gestaltung sozialer Interaktionen beziehen. Angehörige legen neben dem Kriterium »Wohlfühlen« besonderen Wert auf die Qualität der Versorgung (z. B. Förderung der Privatsphäre), das Personal (z. B. Anzahl und Kompetenz der angestellten Fachkräfte) und auf Kriterien bezüglich der Lage und Ausstattung der Institution (vgl. Josat et al. 2006).

\subsection{Studie: „Weiterentwicklung der Qualitätsberichterstattung in der Langzeitpflege»}

Im Folgenden werden Ergebnisse aus der vom AOKBundesverband und dem Zentrum für Qualität in der Pflege (ZQP) geförderten Studie zur »Weiterentwicklung der Qualitätsberichterstattung in der
Langzeitpflege« vorgestellt (vgl. Sonntag et al. 2017). Diese repräsentative Befragung von AOK-versicherten Pflegebedürftigen stellte die Nutzerperspektive in den Mittelpunkt der Betrachtung. Ziel der Studie war es, relevante Kriterien zu identifizieren, die Pflegebedürftige und deren Angehörige bei der Auswahl einer (stationären) Pflegeeinrichtung wirksam unterstützen. Dabei sollten differenzierte Aussagen über die jeweiligen Präferenzen von Betroffenen und Angehörigen sowie von ambulant bzw. stationär versorgten Pflegebedürftigen ermittelt und separat betrachtet werden. Die zuletzt genannte Subgruppenbetrachtung (ambulant vs. stationär) ermöglicht dabei die Identifizierung von Qualitätspräferenzen, die auf tatsächlichen Erfahrungen beruhen. Diese können mit solchen verglichen werden, die auf einer hypothetischen Betrachtung basieren. Diese Studie ergänzt damit vorliegende Untersuchungen (vgl. Geraedts et al. 2012; Josat 2005; Josat et al. 2006; Stemmer und Arnold 2014) und stellt einen weiteren Schritt zur Erfassung der Nutzerperspektive dar.

\section{- Methoden und Studiendesign}

Die quantitative Studie beruhte auf einer postalischen Befragung von AOK-Versicherten, die im Sinne des Pflegeversicherungsgesetzes (SGB XI) als pflegebedürftig eingestuft wurden. Eingeschlossen wurden somit auch Personen, die ausschließlich durch Angehörige versorgt werden und aktuell weder eine ambulante noch eine stationäre Versorgungsleistung in Anspruch nehmen, sondern einzig Pflegegeld beziehen. Das Sampledesign beruhte auf einer disproportional geschichteten Zufallsstichprobe. Als Schichtungsmerkmale fungierten die Art der Versorgung (ambulant/stationär), das Geschlecht sowie die Pflegestufe. Innerhalb der definierten Schichten wurden die Versicherten zu gleichen Anteilen mittels einfacher Zufallsauswahl selektiert (vgl. Schnell et al. 1999). Dies hatte u. a. den Vorteil, auch "schwach besetzte" Teilstichproben (pflegebedürftige Männer der Pflegestufe 3) ohne Bias zu repräsentieren. Um die Disproportionalität in der Stichprobenziehung auszugleichen, wurde ein Gewichtungsfaktor generiert, sodass repräsentative Aussagen für die AOK-versicherten Pflegebedürftigen hinsichtlich der Schichtungsmerkmale gemacht werden können. Die Haupt- 


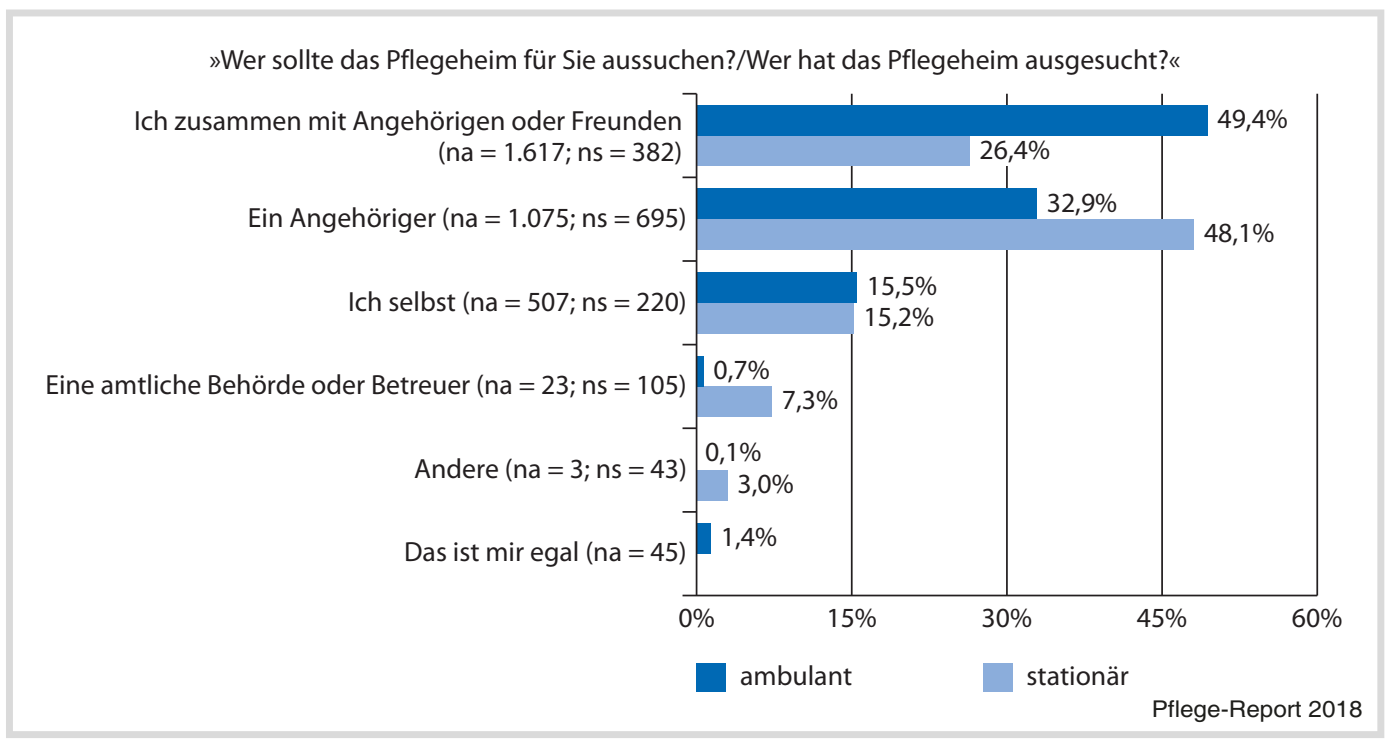

- Abb. 9.1 Akteure bei der Auswahl einer Pflegeeinrichtung (in \%) (Quelle: Sonntag et al. 2017, S. 39)

datenerhebung wurde im Zeitraum von Mitte Februar 2017 bis Ende April 2017 durchgeführt. Für die Teilnahme an der Studie wurden insgesamt 20.608 pflegebedürftige AOK-Versicherte der elf landesweiten AOKs zur Befragung eingeladen. Die Nettostichprobe umfasste 5.021 Fragebögen. Dies entspricht einer Rücklaufquote von 24,4 \%. Die Konzeption des Fragebogens bezog bereits entwickelte Instrumente ein (vgl. ABK 2007; BMFSFJ 2000; IfD Allensbach 2009; Geraedts 2012) und berücksichtigte insgesamt 79 Qualitätskriterien, die sich unter zehn Qualitätsbereiche (Lage und Erreichbarkeit, Wohnen und Ausstattung, Kosten, Essen und Trinken, Pflege, medizinische Versorgung, Freizeitaktivitäten, Wäsche und Reinigung, Lebensführung und Privatsphäre sowie soziale Kontakte) subsumieren lassen. Die Wichtigkeit der Qualitätsbereiche sowie der einzelnen Qualitätskriterien wurde auf einer fünfstufigen Bewertungsskala von »überhaupt nicht wichtig« bis »entscheidend « eingeschätzt.

\section{- Ausgewählte Ergebnisse der Studie: Akteure} bei der Auswahl einer Pflegeeinrichtung

Mit dem Auftreten einer Pflegebedürftigkeit stehen Betroffene sowie deren Angehörige vor der Entscheidung, wie eine adäquate pflegerische Versorgung erbracht werden soll und kann. In diesem $\mathrm{Zu}-$ sammenhang ist es von Interesse, wer die Auswahl einer Pflegeeinrichtung übernimmt. Neben dem Pflegebedürftigen selbst kann dies u. a. durch Angehörige oder gesetzliche Vertreterinnen bzw. Vertreter geschehen (• Abb. 9.1). Von den ambulant Versorgten ( $n=3.270)$ gaben fast die Hälfte $(49,4 \%)$ an, dass die Auswahl gemeinsam mit Angehörigen oder Freunden erfolgen sollte. Ein Drittel (32,9\%) der ambulant versorgten Befragungsteilnehmerinnen und -teilnehmer würden eine Pflegeeinrichtung durch Angehörige aussuchen lassen. Lediglich 15,5 \% möchten die Auswahl selbst vornehmen. Ein sehr geringer Anteil von 0,7\% würde die Suche an einen Betreuer oder eine Betreuerin bzw. an eine gesetzliche Behörde übergeben. Für 1,4 \% der ambulant Befragten spielt es keine Rolle, wer eine geeignete Pflegeeinrichtung aussucht. Unter den stationär Versorgten $(n=1.446)$, die bereits in der Situation waren, eine Pflegeeinrichtung auszuwählen, zeigten sich deutliche Unterschiede: Lediglich ein Viertel $(26,4 \%)$ der stationär Befragten gab an, dass sie eine Pflegeeinrichtung gemeinsam mit Angehörigen oder Freunden aussuchten. Weitere 15,2 \% wählten ihre stationäre Unterbringung selbst aus und in 7,3 \% der Fälle übernahm eine amtliche Behörde resp. der Betreuer oder die Betreuerin das Auswahlverfahren. 3,0 \% benannten »andere Personen«, 


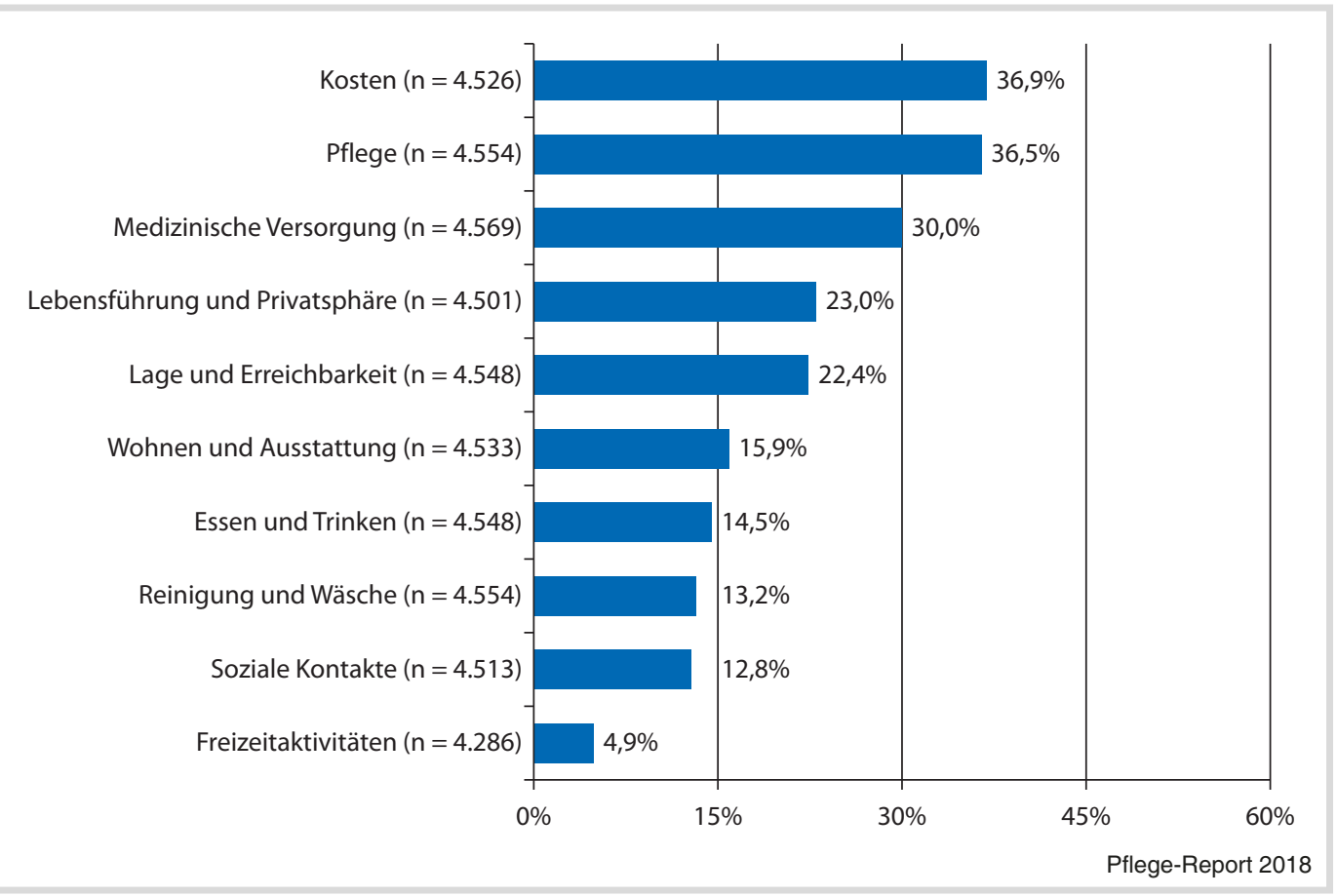

- Abb. 9.2 Entscheidende Qualitätsbereiche (gesamt, in \%) (Anmerkung: Darstellung Antwortkategorie »Entscheidend«) (Quelle: Sonntag et al. 2017, S. 88)

womit zumeist der Sozialdienst eines Krankenhauses gemeint war. So sind es also nicht nur die Betroffenen selbst oder deren Angehörige, die einen Pflegeheimplatz auswählen, sondern zu einem geringen Anteil auch Akteure des Gesundheitswesens. Mehrheitlich $(48,1 \%)$ waren es innerhalb der stationären Versorgungsgruppen jedoch Angehörige, die eine geeignete Pflegeeinrichtung für die Pflegebedürftigen aussuchten. Dies untermauert die Annahme, dass Angehörige eine relevante und zu berücksichtigende Nutzergruppe bei der Auswahl einer Pflegeeinrichtung darstellen. Aus diesem Grund ist eine separate Betrachtung der beiden primären Nutzergruppen (Pflegebedürftige vs. Angehörige) bei der Erfassung relevanter Qualitätskriterien bei der Heimauswahl angebracht.

\section{- Ausgewählte Ergebnisse der Studie: Entscheidende Qualitätsbereiche im Auswahlprozess aus Nutzersicht}

Ökonomische Aspekte spielen bei der Auswahl einer Pflegeeinrichtung eine wesentliche Rolle. Bei den befragten Verbraucherinnen und Verbraucher rangieren Informationen zum Qualitätsbereich Kosten (36,9\%) ganz vorn (• Abb. 9.2). Entscheidender Aspekt ist dabei für die Befragungsteilnehmerinnen und -teilnehmer eine verständliche und nachvollziehbare Übersicht über die Gesamtkosten einer Pflegeeinrichtung. In der Rangfolge an zweiter Stelle folgt der Bereich Pflege (36,5\%), der neben der pflegerischen Versorgung auch personalbezogene Kriterien beinhaltet. Eine hohe Bewertung innerhalb dieses Qualitätsbereichs erzielen Kriterien wie bspw. ein respektvoller Umgang des Pflegepersonals gegenüber dem Pflegebedürftigen, eine sorgfältig durchgeführte und an die Bedürfnisse angepasste Pflege sowie ein ausreichender Personalschlüssel. Auf Platz drei der Präferenzliste befindet sich der Qualitätsbereich medizinische Versorgung (30,0\%), der als wichtig erachtete Kriterien wie die Versorgung durch Haus- und Fachärzte vor Ort im Pflegeheim sowie die Einbeziehung von Angehörigen bei medizinischen Entscheidungen beinhaltet. Informationen, die eine autonome Lebensführung 


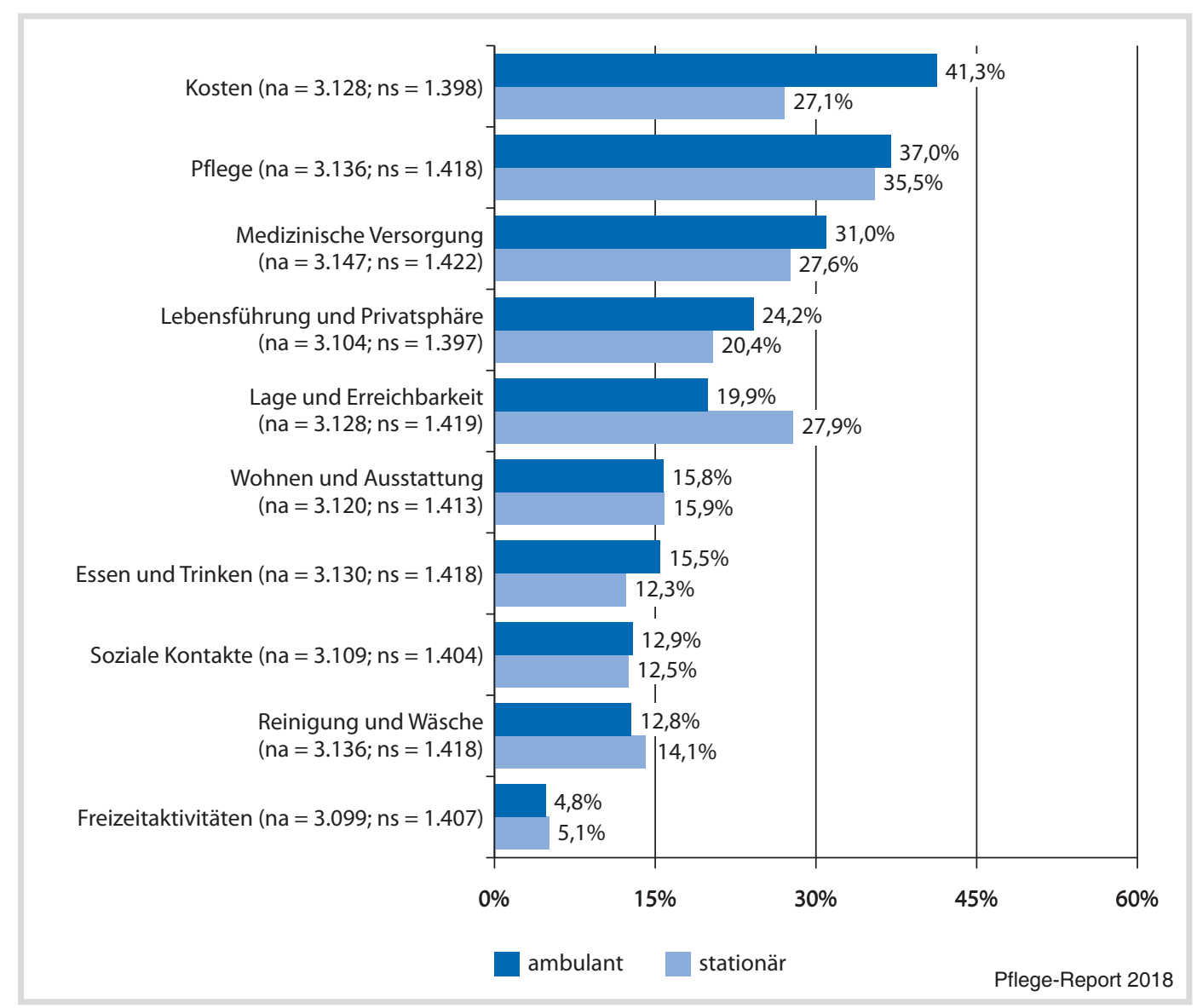

- Abb. 9.3 Entscheidende Qualitätsbereiche nach Versorgungsart (in \%) (Anmerkung: Darstellung Antwortkategorie »Entscheidend«) (Quelle: Sonntag et al. 2017, S. 90)

und Privatsphäre $(23,0 \%)$ betreffen, sind in der Rangfolge an vierter Stelle angesiedelt. Entscheidende Kriterien für die Auswahl einer Pflegeeinrichtung sind hierbei die Möglichkeit, zwischen Einzel- oder Zweibettzimmer zu wählen und auf die Auswahl eines Mitbewohners (bei Zwei- und Mehrbettzimmern) Einfluss zu nehmen. Den fünften Rang nimmt der Qualitätsbereich Lage und Erreichbarkeit (22,4 \%) ein. Hierunter fallen Kriterien wie die Nähe zum ursprünglichen Wohnort sowie zu Freunden und Angehörigen. Mit etwas Abstand sind auf den unteren Rängen die Qualitätsbereiche Wohnen und Ausstattung (15,9\%), Essen und Trinken (14,5\%), Reinigung und Wäsche (13,2\%) sowie soziale Kontakte $(12,8 \%)$ vorzufinden. Im Verhältnis zu den anderen Bereichen wird der Qualitäts- bereich Freizeitaktivitäten (4,9\%) deutlich seltener als »entscheidend « bewertet.

Eine differenzierte Betrachtung der Präferenzen für die verschiedenen Qualitätsbereiche hinsichtlich der Subgruppen »Art der Versorgung « (ambulant/stationär) und »Befragungspersonen « (Pflegebedürftiger/Angehöriger) zeichnet folgendes Bild:

Die Betrachtung der Teilgruppen, die bereits ein Pflegeheim ausgewählt haben (stationär), und jenen, die möglicherweise in Zukunft eines aussuchen werden (ambulant), weist sowohl Unterschiede in der Rangfolge gegenüber der Gesamtbetrachtung als auch in der Gegenüberstellung auf. Informationen zur Pflege (35,5\%), Lage und Erreichbarkeit (27,9\%) sowie zur Medizinischen Versorgung $(27,6 \%)$ rangieren unter stationär Ver- 


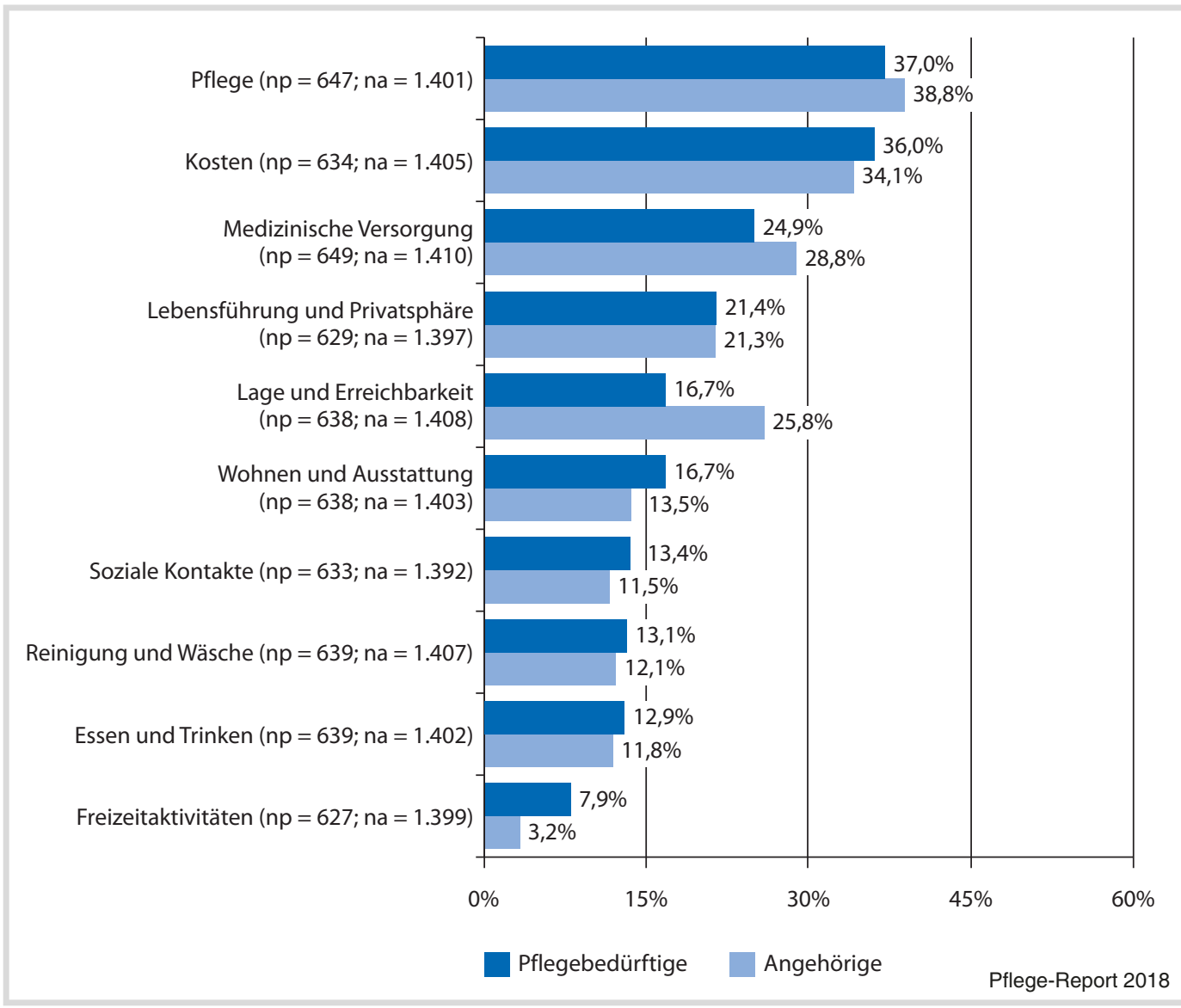

- Abb. 9.4 Entscheidende Qualitätsbereiche nach Befragungsperson (in \%) (Anmerkung: Antwortkategorie »Entscheidend«; Befragungsteilnehmer, die den Fragebogen gemeinsam ausfüllten, werden nicht dargestellt) (Quelle: Sonntag et al. 2017, S. 91)

sorgten auf den Plätzen eins bis drei. Unter den ambulant Versorgten sind wie in der Gesamtbetrachtung die ersten drei Plätze mit den Qualitätsbereichen Kosten (41,3\%), Pflege (37,0\%) und Medizinische Versorgung (31,0\%) besetzt. Statistisch signifikante Bewertungsunterschiede $(\mathrm{p}<0,05)$ zwischen ambulant und stationär Versorgten lassen sich für die folgenden Qualitätsbereiche feststellen: Die Bewertung des Qualitätsbereichs Lage und Erreichbarkeit weist höchst signifikante Unterschiede zwischen den zwei Versorgungsarten auf: Für bereits stationär versorgte Personen $(27,9 \%)$ sind Informationen für diesen Qualitätsbereich deutlich entscheidender bei der Auswahl einer Pflegeeinrichtung als für ambulant Versorgte (19,9\%). Hinsichtlich des Bereichs Essen und Trinken zeigen sich gleichfalls deutliche Unterschiede zwischen den beiden Versorgungsgruppen. Etwas unerwartet räumen ambulant Versorgte (15,5 \%) den Informationen zu Essen und Trinken mehr Priorität ein als stationär versorgte Personen (12,3\%). Auch Informationen zur medizinischen Versorgung werden von ambulant versorgten Personen $(31,0 \%)$ gegenüber stationär versorgten Personen $(27,6 \%)$ häufiger genannt. Signifikante Unterschiede zeigen sich gleichfalls in der Bewertung der Informationen über die Kosten einer Pflegeeinrichtung. Während $41,3 \%$ der ambulanten Versorgungsgruppen Informationen zu den Kosten als entscheidend bei der Auswahl einer Pflegeeinrichtung bewerten, sind es innerhalb der stationären Gruppen nur 27,1\%. Signifikant unterschiedliche Bewertungen weisen 
Tab. 9.1 Die zehn am höchsten bewerteten Kriterien bei der Auswahl einer Pflegeeinrichtung

\begin{tabular}{|l|l|l|}
\hline Kriterium & Qualitätsbereich & $\begin{array}{l}\text { Mittelwert } \\
(\text { Standardabweichung) }\end{array}$ \\
\hline $\begin{array}{l}\text { Respektvolles Verhalten des Pflegepersonals } \\
\text { gegenüber dem Pflegebedürftigen }\end{array}$ & Pflege (Personal) & $4,27(0,592)$ \\
\hline $\begin{array}{l}\text { Gutes Beschwerdemanagement } \\
\text { Eine sorgfältig durchgeführte Pflege }\end{array}$ & Lebensführung und Privatsphäre & $4,25(0,635)$ \\
\hline Gut ausgebildetes Pflegepersonal & Pflege & $4,22(0,620)$ \\
\hline $\begin{array}{l}\text { Ausreichender Pflegepersonalschlüssel } \\
\text { Bedürfnisorientierte Pflege }\end{array}$ & Pflege (Personal) & $4,21(0,656)$ \\
\hline Dass sich viel Zeit für die Pflege genommen wird & Pflege (Personal) & $4,21(0,614)$ \\
\hline $\begin{array}{l}\text { Verständliche und nachvollziehbare Übersicht } \\
\text { über die Gesamtkosten }\end{array}$ & Kflege & $4,19(0,631)$ \\
\hline $\begin{array}{l}\text { Möglichkeit, ungestört zu sein } \\
\text { Guter Kontakt zu den Pflegekräften }\end{array}$ & $4,18(0,613)$ \\
\hline
\end{tabular}

die Versorgungsgruppen zudem hinsichtlich des Qualitätsbereichs Lebensführung und Privatsphäre auf. Hierzu präferieren $24,2 \%$ der ambulant gegenüber 20,4 \% der stationär Versorgten Informationen (- Abb. 9.3).

Im Hinblick auf die Präferenzsetzung von Qualitätsbereichen zwischen Angehörigen und Pflegebedürftigen zeigen sich keine Unterschiede in der Rangfolge. Auf Platz eins rangiert der Qualitätsbereich Pflege, gefolgt von Kosten und der Medizinischen Versorgung. Auffällig und statistisch signifikant $(p<0,05)$ ist der Bewertungsunterschied zwischen den zwei Befragungsgruppen hinsichtlich des Qualitätsbereichs Lage und Erreichbarkeit. Mehr als ein Viertel (25,8\%) der Angehörigen nennen diesen Bereich als entscheidend im Auswahlprozess, im Vergleich zu 16,7 \% der pflegebedürftigen Personen. Weitere statistisch signifikante Unterschiede in der Bewertung zwischen Angehörigen und Pflegebedürftigen können für die Qualitätsbereiche Wohnen und Ausstattung, Freizeitaktivitäten sowie Pflege nachgewiesen werden. Informationen zu dem Bereich Wohnen und Ausstattung werden von den Betroffenen selbst (16,7 \%) im Vergleich zu deren Angehören (13,5\%) häufiger als »entscheidend « bei der Auswahl einer Pflegeeinrichtung eingeschätzt. Einen ebenfalls signifikant höheren Stel- lenwert nehmen für die Pflegebedürftigen (7,9\%) Freizeitaktivitäten im Gegensatz zu den Angehörigen $(3,2 \%)$ ein. Insgesamt ist der Qualitätsbereich Freizeitaktivitäten jedoch in beiden Befragungsgruppen der am wenigsten relevante Bereich, zu welchem Informationen als entscheidend bewertet werden. Am häufigsten als »entscheidend «, aber dennoch signifikant unterschiedlich, bewerteten Angehörige und Pflegebedürftige den Qualitätsbereich Pflege (• Abb. 9.4).

\section{- Ausgewählte Ergebnisse der Studie: Die} zehn am höchsten bewerteten Kriterien bei der Auswahl einer Pflegeeinrichtung

Die zehn Qualitätsbereiche (Lage und Erreichbarkeit, Wohnen und Ausstattung, Kosten, Essen und Trinken, Pflege, medizinische Versorgung, Freizeitaktivitäten, Wäsche und Reinigung, Lebensführung und Privatsphäre sowie soziale Kontakte) wurden durch insgesamt 79 Kriterien abgebildet. Für die Analyse wurden Mittelwerte für jedes Qualitätskriterium berechnet. Je höher der Mittelwert, desto wichtiger war/ist das jeweilige Kriterium für die Befragten bei der Auswahl einer Pflegeeinrichtung. - Tab. 9.1 zeigt die zehn wichtigsten Kriterien, die jenseits der übergeordneten Rangfolge der Qualitätsbereiche aus Sicht aller Befragten bei der Aus- 
wahl einer Pflegeeinrichtung zentral sind. Sechs dieser zehn wichtigsten Kriterien sind dem Qualitätsbereich Pflege zuzuordnen und betreffen die sozialen und fachlichen Kompetenzen des Pflegepersonals, den Personalschlüssel, das für die Pflege zur Verfügung stehende Zeitbudget sowie die Pflegequalität. Hervorzuheben ist die Bedeutsamkeit des Kriteriums, dass adäquat auf Beschwerden reagiert werden sollte. Die Möglichkeit, sich zu beschweren, ist hier als ein partizipatives Instrument zu werten.

\subsection{Fazit}

Zusammenfassend betrachtet wird deutlich, dass das Thema Pflegequalität resp. Qualitätsberichterstattung aus Sicht von Nutzerinnen und Nutzern relevant ist und an Bedeutung gewinnt. Zur Verbesserung der Qualitätsberichterstattung ist es erforderlich, sowohl die Experten- als auch die Nutzerperspektive zu kennen und einzubeziehen, um eine fundierte Qualitätsbeurteilung zu erzielen und die Qualität in der Pflege effektiv zu verbessern. Da die Sicht Betroffener häufig von jener der Expertinnen und Experten abweicht, ist das Verständnis des konkreten Erfahrungswissens der Nutzerinnen und Nutzer sowie ihrer spezifischen Bedürfnisse und Erwartungen für die Qualitätsbeurteilung und -weiterentwicklung von grundlegender Bedeutung (vgl. Bolz 2016). Darüber hinaus ist die Kenntnis von Qualitätskriterien erforderlich, die für potenzielle Nutzerinnen und Nutzer relevant sein könnten. Erst so kann gewährleistet werden, dass nutzerfreundlich aufbereitete Informationen den Entscheidungsprozess bei der Auswahl einer stationären Pflegeeinrichtung unterstützen. Die vorgestellte Studie liefert vielschichtige Erkenntnisse hinsichtlich relevanter Qualitätskriterien in der Langzeitpflege aus differenzierter Nutzersicht, nämlich aus Sicht der Betroffenen, von deren Angehörigen sowie aus Sicht ambulant oder stationär versorgter Pflegebedürftiger.

Die Sichtweise der Angehörigen ist bedeutend, denn diese nehmen oftmals eine zentrale Rolle bei der Suche, Evaluation und Auswahl einer adäquaten Pflegeeinrichtung ein. Sie beeinflussen den Entscheidungsprozess meist maßgeblich resp. treffen die Entscheidung, wie die Ergebnisse dieser Studie bestätigen. Im Vergleich zu anderen Studien (vgl. Geraedts et al. 2012; Josat 2005; Curry und Stark 2000) fällt die Divergenz der Präferenzsetzung einzelner Qualitätsbereiche zwischen den beiden Nutzergruppen »Angehörige« und »Pflegebedürftige« allerdings eher gering aus, wobei sich punktuell Bewertungsunterschiede festhalten lassen. So rangieren die Qualitätsbereiche »Pflege«, »Kosten « und »Medizinische Versorgung " in beiden Untergruppen auf den Plätzen eins bis drei. Jedoch nimmt bspw. der Qualitätsbereich "Lage und Erreichbarkeit« für Angehörige einen deutlich höheren Stellenwert ein als für die Betroffenen selbst. Für die künftige Qualitätsberichterstattung gilt es, diese Differenzen zu berücksichtigen und Informationsinhalte auf die spezifischen Bedarfe und Wünsche Angehöriger und Betroffener zuzuschneiden. Entsprechend der Präferenzsetzung der einzelnen Qualitätsbereiche ist bei der künftigen Berichterstattung speziell darauf zu achten, detaillierte Informationen über die Kosten resp. eine transparente Kostenaufschlüsselung zu geben sowie die Kriterien, die die pflegerische und medizinische Versorgung betreffen, ins Zentrum der Informationsvermittlung zu stellen. Beispielsweise ist die Zurverfügungstellung von Informationen hinsichtlich der Personalausstattung wie eine detaillierte Übersicht des Personalschlüssels für Angehörige sowie Pflegebedürftige besonders wichtig.

Neben den Präferenzsetzungen stratifiziert nach Angehörigen und Pflegebedürftigen liefert die Studie Erkenntnisse zu den Befragungsgruppen nach der Art der Versorgung. Es galt zu untersuchen, inwiefern die Gewichtung der einzelnen Qualitätsbereiche je nach aktuellem Erfahrungshorizont differiert. Während stationär Versorgte die Qualitätskriterien aufgrund ihrer Erfahrungswerte einschätzten, mussten sich die ambulant Versorgten in ein potenziell eintreffendes Szenario versetzen. In diesen Subgruppen zeigen sich im Vergleich zu Pflegebedürftigen und Angehörigen deutlichere Unterschiede in der Gesamtbewertung der Qualitätsbereiche. So ist ambulant Versorgten die Klärung von Kostenaspekten am dringlichsten, gefolgt von der »Pflege«, der »medizinischen Versorgung» sowie der "Lebensführung und Privatsphäre«. Für die befragten Heimbewohnerinnen und -bewohner 
hat die "pflegerische Versorgung" höchste Relevanz. Danach folgen für die stationär Versorgten die Qualitätsbereiche »Lage und Erreichbarkeit«, »Medizinische Versorgung « sowie »Kosten«. Die Differenz zwischen den Angaben der stationär und ambulant Versorgten könnte mit höheren Autonomieressourcen seitens der ambulant Gepflegten erklärt werden. Des Weiteren werden Entscheidungen für die Wahl einer Pflegeeinrichtung häufig unter Handlungsdruck getroffen. Dementsprechend sollte die Pflegeberichterstattung (potenzielle) Nutzerinnen und Nutzer frühzeitig erreichen, sodass ausreichend Zeit zur Vorbereitung sowie die Möglichkeit zur Teilhabe am Auswahlprozess bleiben.
Insgesamt weisen die Resultate der vorgestellten Studie darauf hin, dass Informationsangebote eine große Bandbreite an Inhalten abdecken sollten und diese entsprechend der Priorisierung der einzelnen Qualitätsbereiche sowie ausgerichtet an die verschiedenen Adressatengruppen strukturiert und kategorisiert werden sollten. Somit können die differenten Nutzergruppen während des Entscheidungsprozesses angesichts ihrer individuellen Bedürfnisse wirksam gestärkt werden, adäquate Entscheidungen getroffen und die Zufriedenheit mit dieser Auswahl möglicherweise erhöht werden. Dies wiederum mag langfristig Einfluss auf die Qualität in der Langzeitpflege nehmen.

Open Access Dieses Kapitel wird unter der Creative Commons Namensnennung 4.0 International Lizenz (http://creativecommons. org/licenses/by/4.0/deed.de) veröffentlicht, welche die Nutzung, Vervielfältigung, Bearbeitung, Verbreitung und Wiedergabe in jeglichem Medium und Format erlaubt, sofern Sie den/die ursprünglichen Autor(en) und die Quelle ordnungsgemäß nennen, einen Link zur Creative Commons Lizenz beifügen und angeben, ob Änderungen vorgenommen wurden.

Die in diesem Kapitel enthaltenen Bilder und sonstiges Drittmaterial unterliegen ebenfalls der genannten Creative Commons Lizenz, sofern sich aus der Abbildungslegende nichts anderes ergibt. Sofern das betreffende Material nicht unter der genannten Creative Commons Lizenz steht und die betreffende Handlung nicht nach gesetzlichen Vorschriften erlaubt ist, ist für die oben aufgeführten Weiterverwendungen des Materials die Einwilligung des jeweiligen Rechteinhabers einzuholen.

\section{Literatur}

Arbeitsgemeinschaft Berliner Koordinierungsstellen (ABK) (2007) Rund ums Alter. Verbraucherinformationen. Nr.12 Checkliste Pflegeheim. https://www.evangelischesjohannesstift.de/sites/default/files/altenhilfe/pdf/ Informationsblaetter/Nr.12ChecklistePflegeheim.pdf. Zugegriffen: 28. November 2017

Bolz H (2016) Bedeutung und Ziele einer Befragung von Pflegekunden. Aus: Befragung von Pflegekunden. Zielsetzung - Inhalte - Planung - Umsetzung. Springer, Wiesbaden

Bundesministerium für Familie, Senioren, Frauen und Jugend (2000) Auf der Suche nach einem Heim. Leitfaden zur Wahl eines Pflegeplatzes

Curry A, Stark S (2000) Quality of service in nursing homes. Health services management research 13(4):205-215

Donabedian A. (1966) Evaluating the quality of medical care. The Milbank memorial fund quarterly 44(3):166-206

Duffy J, Duffy M, Kilbourne W (2001) A comparative study of resident, family and administrator expectations for service quality in nursing homes. Health Care Management Review 26(3):75-85

Engels D, Pfeuffer F (2007) Die Einbeziehung von Angehörigen und Freiwilligen in die Pflege und Beutreuung in
Einrichtungen - Forschungsprojekt des Bundesministeriums für Familie, Senioren, Frauen und Jugend »Möglichkeiten und Grenzen einer selbsständigen Lebensführung in Einrichtungen«. Institut für Sozialforschung und Gesellschaftspolitik, Köln

Geraedts M, Brechtel T, Zöll R, Hermeling P (2012) Beurteilungskriterien für die Auswahl einer Pflegeeinrichtung. In: Böcken J, Braun B, Repschläger U (Hrsg) Gesundheitsmonitor 2011. Bürgerorientierung im Gesundheitswesen. Kooperationsprojekt der Bertelsmann Stiftung und der BARMER GEK, S 155-172. Verlag Bertelsmann Stiftung, Gütersloh

Hasseler M (2014) Heraus- und Anforderungen an eine systematische Qualitätsmessung und -berichterstattung in der Langzeitpflege. Vierteljahrs Hefte zur Wirtschaftsforschung, Duncker \& Humblot, 83(4):67-85

Hibbard J, Peters E (2003) Supporting Informed Consumer Health Care Decision: Data Presentation Approaches that Facilitate the Use of Information in Choice. Annual Review of Public Health 24:413-433

IfD Allensbach (2009) Pflege in Deutschland. Ansichten der Bevölkerung über Pflegequalität und Pflegesituation. Ergebnisse einer Repräsentativbefragung. http://www. mk-kliniken.de/pdf/presse/Allensbach-Studie.pdf. Zugegriffen: 16. Oktober 2017 
Josat S (2005) Welche Qualitätskriterien sind Angehörigen in der stationären Altenpflege wichtig? Eine Einzelfallstudie. Pflege 18(3):169-175

Josat S, Schubert HJ, Schnell MW, Köck C (2006) Qualitätskriterien, die Altenpflegeheimbewohnern und Angehörigen wichtig sind - Eine Literaturanalyse. Pflege 19(2):79-87

Kämmer K (2008) Altenhilfe in einer Gesellschaft des langen Lebens Pflegemanagement in Altenpflegeeinrichtungen (Vol 5). Schültersche Verlagsgesellschaft mbH \& CoKg, Hannover

Kämmer K (2015) Pflegemanagment in Altenpflegeeinrichtungen - Zukunftsorientiert führen, konzeptionell steuern, wirtschaftlich lenken (Vol. 6). Schültersche Verlagsgesellschaft $\mathrm{mbH} \&$ CoKg, Hannover

Kelle U, Metje B, Niggemann C (2014) Datenerhebung in totalen Institutionen als Forschungsgegenstand einer kritischen gerontologischen Sozialforschung. In: Amann A, Kolland F (Hrsg) Das erzwungene Paradies des Alters? Weitere Fragen an eine Kritische Gerontologie (Vol. 2). Springer VS, Wiesbaden, S 175-206

Klingenfeld H (1999) Heimübersiedlung und Lebenszufriedenheit älterer Menschen. Person- und Umweltfaktoren und ihr Einfluss auf die Anpassungsleistung an das Heimleben. Europäische Hochschulschriften, Reihe VI Psychologie, Bd 643. Europäischer Verlag der Wissenschaften, Frankfurt am Main

Mittnacht B (2010) Qualitätsentwicklung und Nachhaltigkeit im Kontext häuslicher Pflegearrangements: Entwicklungstrends und Perspektiven. Jacobs Verlag Düsseldorf

Nübling R, Kriz D, Kress G, Schrempp C, Löschmann C, Schmidt J (2004) Angehörigenbefragungen - Potentiale für das interne Qualitätsmanagement in Altenpflegeeinrichtungen. Gesundheitswesen 66:380-386

Rantz MJ, Zwygart-Stauffacher M, Popejoy L, Grando VT, Mehr DR, Hicks LL, Bostick J (1999) Nursing home care quality: A multidimensional theoretical model integrating the views of consumers and providers. Journal of nursing care quality 14(1):16-37

Roth G (2002) Qualität in Pflegeheimen. Forschungsgesellschaft für Gerontologie e.V. Expertise im Auftrag des Bundesministeriums für Familie, Senioren, Frauen und Jugend. Universität Dortmund, Institut für Gerontologie

Schenk L, Meyer R, Behr A, Kuhlmey A, Holzhausen M (2013) Quality of Life in Nursing Homes: Results of a Qualitative Resident Survey. Quality of Life Research 22:2929-2938

Schnell R, Hill PB, Esser E (1999) Methoden der empirischen Sozialforschung. 6. Aufl. Oldenbourg, München

Sonntag PT, Baer NR; Schenk L (2017) Weiterentwicklung der Qualitätsberichterstattung in der Langzeitpflege. Eine quantitative Versichertenbefragung. Endbericht für das ZQP und den AOK-Bundesverband. Zentrum für Qualität in der Pflege und AOK-Bundesverband, Berlin. https:// www.zqp.de/wp-content/uploads/ZQP_Abschlussbericht_Qualitätskriterien.pdf
Strotbek J, Etgeton S, Palmowski S, Schuhen A, Schmuhl M (2017) Reformkonzept Verbraucher/ -innenorientierte Qualitätsberichterstattung in der Pflege - Anforderungen und Lösungsvorschläge mit besonderem Blick auf Lebensqualität, Personalausstattung und Erfahrungswissen. Weiße Liste, Bertelsmann Stiftung, Gütersloh

Stemmer R, Arnold J (2014) Expertise zur Eignung von Indikatoren zur Messsung und Darstellung von Ergebnisqualität in der stationären Pflege im Bereich der sozialen Pflegeversicherung. IKJ ProQualitas GmbH, Mainz

Walter H (1995) Das Alter leben! Herausforderungen und neue Lebensqualitäten. Wissenschaftliche Buchgesellschaft, Darmstadt

Wingenfeld K, Engels D, Engel H, Franz S, Kleina T, Mehlan S (2011) Entwicklung und Erprobung von Instrumenten zur Beurteilung der Ergebnisqualität in der stationären Altenhilfe, Bielefeld und Köln 